\title{
Circuit
}

Musiques contemporaines

\section{Hugh Le Caine, de la saqueboute au multi-track}

\section{Gayle Young}

Volume 19, numéro 3, 2009

Pionniers canadiens de la lutherie électronique

URI : https://id.erudit.org/iderudit/038255ar

DOI : https://doi.org/10.7202/038255ar

Aller au sommaire du numéro

Éditeur(s)

Les Presses de l'Université de Montréal

ISSN

1183-1693 (imprimé)

1488-9692 (numérique)

Découvrir la revue

Citer cet article

Young, G. (2009). Hugh Le Caine, de la saqueboute au multi-track. Circuit, 19(3), 9-37. https://doi.org/10.7202/038255ar

\section{Résumé de l'article}

Dans cet extrait de son livre Blues pour saqueboute, Gayle Young retrace les premiers pas de la musique électronique et rappelle une période spécifique durant laquelle les innovations scientifiques et artistiques étaient fortement encouragées par le public et les institutions publiques. L'article est bâti à partir de nombreuses entrevues avec des collègues de Hugh Le Caine et des extraits de correspondances. Les possibilités musicales des instruments de Le Caine sont soulignées dans le livre dont proviennent ces extraits, de même que leur fonctionnement technique. L'intérêt de Le Caine pour le clavier à touches sensibles et pour les contrôles simultanés de différents paramètres musicaux est déjà évident dans la construction du saqueboute de 1948, un instrument pour lequel il utilisait le contrôle de voltage pour la première fois, construisant ainsi le premier synthétiseur analogique. Sa composition Dripsody (1955), élaborée sur un prototype multipiste, utilisait un clavier similaire à celui du saqueboute, mais plutôt que de contrôler des générateurs audio électroniques, il contrôlait la vitesse de lecture de plusieurs bobines de ruban. Pour Le Caine, le son est l'élément de base de la musique, qu'il soit électronique ou échantillonné.
Tous droits réservés (C) Les Presses de l’Université de Montréal, 2009
Ce document est protégé par la loi sur le droit d'auteur. L’utilisation des services d'Érudit (y compris la reproduction) est assujettie à sa politique d'utilisation que vous pouvez consulter en ligne.

https://apropos.erudit.org/fr/usagers/politique-dutilisation/ 


\section{Hugh Le Caine, de la saqueboute au multi-track ${ }^{1}$}

Gayle Young

\section{Le premier synthétiseur}

La Deuxième Guerre mondiale fut pour les Canadiens le début d'une période de confiance et d'innovation sans précédent. Horace Aubrey la résume ainsi: "Nous pensions que, en tant que Canadiens, nous pouvions réaliser n'importe quoi. Ce fut une époque exaltante à vivre. Elle dura vingt $a^{2} s^{2}$.» Dans les mois qui suivirent la fin de la guerre, le CNRC (Conseil national de recherches du Canada) entreprit de nombreux projets dans le cadre desquels la technologie du radar à faisceaux hertziens fut adaptée à des applications pacifiques. [...]

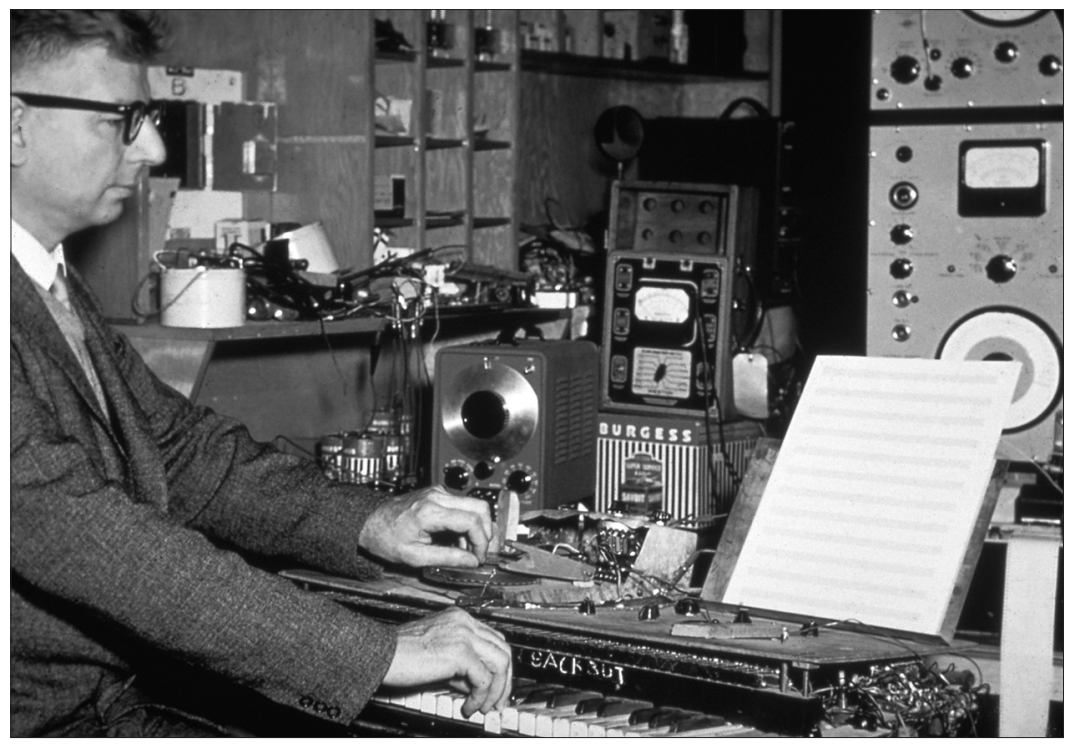

Hugh Le Caine faisant la démonstration de la saqueboute de 1945-48. Ottawa, vers 1954.

(C) Conseil national de recherches Canada. Reproduite avec la permission du ministre des Travaux publics et Services gouvernementaux Canada (2009). Source: Bibliothèque et Archives Canada/ Fonds Hugh Le Caine.
1. Extraits de Gayle Young, Blues pour saqueboute: Hugh Le Caine pionnier de la musique électronique, Ottawa, Musée national des sciences et de la technologie, 1989. ISBN 0-660-12006-2. Publiés avec l'aimable autorisation de l'éditeur. Voir aussi le site $<$ www.hughlecaine.com>. 2. Entrevue, Horace Aubrey. 
3. Entrevue, Lorne Bradley.
Entre 1945 et 1948, Hugh Le Caine (1914-1977) joua le rôle de personneressource au CNRC. Il était toujours disponible lorsqu'on avait besoin de ses services, et a dépanné de nombreux projets. Il discutait souvent en profondeur d'un problème particulier avec un collègue. Si un circuit ne fonctionnait pas comme on l'aurait souhaité, il examinait attentivement tous ses composants et ses tubes. Il discutait de chaque pièce et, au cours de la discussion, le problème devenait soudain évident et était souvent résolu surle-champ. [...]

Aux dires de son ami et collaborateur Lorne Bradley, Le Caine avait une «journée à deux cycles». Il justifiait cet horaire par la théorie suivante: si une personne travaille huit heures par jour, c'est toujours pendant les quatre premières heures qu'elle est le plus efficace. Après quatre heures de travail, une personne ne peut plus fournir un rendement optimal; par conséquent, l'étirement de la période de travail au-delà d'une durée de quatre heures n'est pas la meilleure manière de rentabiliser son temps. Le Caine organisait donc son travail en une «journée à deux cycles» afin d'augmenter son rendement. Au lieu de se conformer à un cycle normal de huit heures de travail, huit heures d'activités personnelles et huit heures de sommeil, il travaillait pendant quatre heures, puis passait quatre heures à ses propres projets, et dormait quatre heures. Cependant, le total n'était pas toujours de douze heures. Il était souvent plus près de treize heures, et Le Caine arrivait donc au laboratoire à des heures variables. Lorsqu'il était en phase avec le reste de la société, il travaillait et bavardait avec les autres au laboratoire; toutefois, après quelques jours à ce rythme, il était déphasé et on ne le voyait plus pendant un certain temps. Ce type d'horaire lui convenait très bien et lui permit d'accrôtre la qualité de ses réalisations3. [...]

Le Caine travailla à plusieurs projets au CNRC et s'occupa intensivement de musique électronique. Il installa un studio chez lui en 1945. Il avait pu louer l'une des maisons de guerre du CNRC à la station de recherche pour 22,30 \$ par mois. Ces maisons présentaient de nombreux inconvénients du fait de leur construction économique, et il était facile d'en louer une. Pour Le Caine cependant, la maison était idéale :

Il y avait beaucoup d'espace. Je pouvais me permettre d'utiliser une pièce entière comme studio d'électronique et une autre comme pièce d'enregistrement. La «salle de concert» abritait tous les instruments que je possédais: piano, harmonium, orgue électronique (celui que j'avais fabriqué à Queen's), violon, guitare, quelques percussions et le modèle en cours d'instrument électronique expérimental. Les appareils d'enregistrement étaient logés dans une pièce séparée, de sorte que le bruit de la machine ne risquait pas d'être capté par le microphone ouvert. Je possédais des blocs d'alimentation et des haut-parleurs en double, ce qui me permettait d'enregistrer 
les instruments expérimentaux en direct si je le désirais. Je disposais de différents moyens d'accroître la réverbération de la pièce. J'avais des dispositifs de démarrage à distance et de protection pour l'enregistreur sur disque et les lectures multiples. Tous ces appareils étaient destinés à simplifier l'enregistrement multiple et permettre à l'interprète de se concentrer sur son jeu. L'ensemble des dispositifs d'enregistrement avait pour fin, quant à lui, de développer un nouvel instrument.

Mon laboratoire d'électronique était situé dans une autre pièce et était équipé d'un oscilloscope, de voltmètres, d'oscillateurs d'essai, de filtres, etc. Cette pièce me servait également de chambre à coucher. C'est là que les nouveaux instruments étaient construits, modifiés et mis à l'essai. Mon équipement consistait en un enregistreur à disque d'acétate qui creusait, à l'aide d'un saphir extrêmement poli, un sillon modulé latéralement par le son dans un disque en aluminium ou en verre recouvert d'une couche d'acétate.

J'ai découvert ce que l'on appelle maintenant la «réverbération de bande » sur mon appareil à disque d'acétate. Cela signifie que très peu de temps après l'enregistrement (30 millisecondes ou plus), celui-ci est rejoué et amené à nouveau sous le burin. C'était très excitant de constater que cela était possible, et j'étais tout à fait surpris par le résultat, car peu de gens avaient fait un usage expérimental ou «libre» de l'enregistreur à disque d'acétate. Le résultat de la rétroaction électronique était fascinant à l'époque et était d'une grande utilité pour les effets spéciaux. J'étais en train d'enregistrer une scène de foule et j'ai utilisé cette méthode pour ajouter des effets d'accumulation. Il a fallu attendre de nombreuses années avant que cette technique ne devienne un cliché, lorsque l'enregistrement sur bande magnétique est devenu courant.

En 1945, je me suis mis à expérimenter des idées auxquelles je pensais depuis quelque temps, des idées qui s'articulaient autour des «belles sonorités» que je n'avais jamais pu produire lors de mes expériences à l'école secondaire. Durant mes années à l'université, ma pensée s'était davantage orientée vers la description de la beauté sonore dans les concepts de la physique.

Dans le cas de mon orgue électronique de 1937, j'étais parvenu aux spécifications requises en formulant clairement ce qui apparaissait à mon oreille comme les défauts les plus marquants du son de l'orgue Hammond (insuffisance de l'attaque et de l'extinction, absence d'un effet de chœur réel et adéquat). La synthèse des harmoniques à partir de la gamme tempérée me semblait une bonne caractéristique du son Hammond, et je l'avais retenue. J'avais finalement estimé que les anches commandées en tension constituaient le meilleur moyen de parvenir aux qualités que je recherchais. Mes expériences éprouvèrent certaines difficultés qui, heureusement, purent être surmontées.

Avec le temps, j'ai compris les limites du terme «beauté», qui revenait fréquemment lorsque l'on discutait des critères de succès d'une œuvre d'art ou de la sonorité d'un instrument de musique au XIXe siècle. Il me semblait que l'on pouvait dire des instruments électroniques existants, pour les comparer à leur équivalent dans un orchestre classique, qu'ils avaient un son mécanique ou qu'ils produisaient des sons sans intérêt, c'est-à-dire qu'ils étaient incapables de transmettre un message 
4. Le Caine, "Recherches au temps perdu: Some personal Recollections of My Work in Electronic Music Written at the Request of a History student, Queen's University", inédit, 1966, p. 27-31.

5. Le Caine, "Electronic Music Today", (inédit, 1966)

6. Le Caine, "A Touch-Sensitive Keyboard fot the Organ", Canadian Music Journal, vol. 3, $\mathrm{n}^{\circ}$ 3, Toronto, Canadian Music Council, Printemps 1959, p. 32. musical, particulièrement en ce qui a trait au détail et à la nuance. J'étais en train de rejoindre le point de vue du XX⿳亠口冋口十 siècle. L'idéal statique de beauté était remplacé par la révélation de nouvelles vérités en regard de la tonalité, des sonorités et des formes musicales... L'objectif du concepteur d'instrument devait être de donner à l'interprète la plus grande maîtrise possible des principaux paramètres, sans nécessairement en rendre son apprentissage difficilet.

Le Caine formula ses vues sur ses nouveaux instruments à partir de sa compréhension de l'évolution des instruments de musique électronique depuis le début du siècle. Dans sa description du contexte historique, il commença par Hermann von Helmholtz, le physicien qui publia Théorie physiologique de la musique en 1863.

Grâce à la clarté, et peut-être à la simplification excessive des théories de Helmholtz, et certainement aussi à cause de sa perspicacité, on a été inondé d'inventions musicales utilisant des sonnettes de porte, des alternateurs et des jets de mercure comme moyens de produire des sons musicaux. Un physicien du nom de [William] Duddell fit la démonstration du premier instrument de musique électronique en 189o, lequel utilisait un circuit résonant connecté à un arc à électrodes de charbon. Ses brevets sur le choralcello s'étendirent jusqu'aux années 1930 et couvraient une diversité stupéfiante de techniques de production sonore, lesquelles sembleraient bien peu réalistes de nos jours.

Parmi les nombreux inventeurs, le nom de Thaddeus Cahill se distingue à cause de sa fameuse démonstration du telharmonium, en 1906, utilisant 1500 haut-parleurs (lesquels, bien entendu, n’étaient que des écouteurs téléphoniques améliorés)5...

Quelque temps plus tard, les tubes à vide furent disponibles dans le commerce. Il était ainsi possible de produire des instruments plus simples et moins encombrants. Une faible quantité d'électricité seulement était nécessaire pour actionner les commandes, car le signal lui-même pouvait maintenant être amplifié à l'aide d'amplificateurs à tubes à vide ${ }^{6}$.

Le Caine en décrit ainsi les effets :

Les amplificateurs et les oscillateurs rendus possibles par les tubes à vide ont complètement révolutionné l'univers des appareils électroacoustiques. Les générateurs électriques de Cahill, qui ressemblaient à une énorme centrale électrique, sont devenus les orgues électroniques compactes d'aujourd'hui...

Un nombre impressionnant d'oscillateurs électriques firent leur apparition et des douzaines de brevets d'instruments électroniques furent déposés avant 1920. En fait, avant la fin de la Première Guerre mondiale, en 1917, Lee De Forest avait publié un article de vulgarisation sur les instruments électroniques... À l'instar de nombreuses autres célébrités de l'époque, son département de publicité était plus développé que son département de recherche. Cela peut expliquer certaines des prédictions extravagantes qu'il fit pour les instruments électroniques. Son article donna le ton aux publications futures. En 1929, [Léopold] Stokowski alla jusqu’à prédire que les instruments traditionnels deviendraient désuets dans quelques années7. 
Les instruments les plus connus des années 1920 sont le Theremin et les ondes Martenot. L'interprète du Theremin ne touche jamais son instrument. Le contrôle du son est obtenu par la position des mains de l'interprète par rapport à l'instrument, l'une donnant la hauteur et l'autre le volume. Le Caine le décrit ainsi :

Theremin [Leon Termen] a produit cinq ou six instruments, dont le plus médiocre, appelé le «Theremin ", a immédiatement conquis le public à cause de ses commandes bizarres et peu commodes... Il avait déclaré aux journalistes qu'il avait vaincu «la résistance offerte au jeu par les instruments». Un musicien a décrit de manière plus précise la pratique du Theremin en la rapprochant de celle d'un violon dépourvu de touche dont l'interprète serait en chute libre ${ }^{8} \ldots$

Les ondes Martenot se présentent sous la forme d'un instrument monophonique à clavier, dont le son fondamental est commandé par les touches et les faibles changements de hauteur par des mouvements latéraux des doigts. La main gauche actionne la commande du volume.

Les compositeurs explorèrent de manière approfondie les possibilités des nouveaux instruments. Le sphârophon de Jorg Mager et d'autres instruments furent conçus pour produire les effets spéciaux requis par les compositeurs pour certaines de leurs œuvres. Un grand nombre de pièces novatrices de musique expérimentale furent écrites, témoignant de l'intérêt soutenu des compositeurs pour les nouveaux instruments.

Dans les années 1930 et 1940, la conception des instruments s'orienta vers les instruments électroniques polyphoniques: les nouvelles orgues électroniques. Comparée à celles des instruments des années 1920, leur conception était conservatrice et modelée sur celle de l'orgue traditionnel à tuyaux, avec ses jeux et ses claviers. À la différence des instruments électroniques plus anciens, elles offraient peu de possibilités quant aux changements subtils de sonorité, mais connurent un grand succès commercial car leur coût était peu élevé et leur clavier polyphonique classique était connu de tous.

En 1945, Le Caine entama la construction d'un nouvel instrument de musique électronique afin de mettre ses idées à l'épreuve. L'instrument fut d'abord construit dans les tiroirs d'un bureau, toutes les pièces électroniques étant cachées; il fut par la suite sorti du bureau et devint un instrument à clavier muni de sa propre console9. Le Caine l'appela la «saqueboute électronique ", en référence à l'instrument à vent médiéval qui fut le précurseur du trombone. Il choisit ce nom probablement parce que l'une des caractéristiques de la saqueboute était une commande de hauteur coulissante, mais il en donna une explication différente dans un article descriptif: «Si j’ai choisi le nom d'un instrument complètement disparu [...] c'était pour conférer à son inventeur une certaine immunité vis-à-vis de la critique ${ }^{10}$. »
9. Matthew Hart, "The Sackbut", Weekend Magazine, 16 juin 1973, p. 22-24. En 1945, lorsque M. Le Caine a commencé à travailler chez lui à ce qui allait devenir la saqueboute, l'instrument n'était qu'une commode pleine de fils et de tubes. Si vous saviez dans quel tiroir chercher, vous pouviez produire des sons.

10. Le Caine, "Electronic Music", Proceedings IRE, vol. 44, $\mathrm{n}^{\circ} 22,1956$, p. 467. 
11. Le Caine, transcription d'un enregistrement.

12. Le Caine, "Electronic Music", Op. cit., p. 465 .
Pour venir à bout de ce qu'il percevait comme l'aspect mécanique et sans intérêt de la sonorité des instruments électroniques, Le Caine se préoccupa davantage de leur musicalité. Il essaya de concevoir l'instrument de telle sorte qu'il soit aussi satisfaisant qu'un violon sur le plan musical, qu'il offre la même précision et les mêmes nuances sonores, mais qu'il permette plus de variations et que son apprentissage soit plus facile. Les nuances subtiles des éléments sonores devaient permettre plus d'expressivité et assurer la continuité de la ligne mélodique.

Lorsqu'il présenta le premier enregistrement de sa saqueboute, Le Caine fit le commentaire suivant: «Nombre de personnes pensent que l'onde carrée est fondamentalement déplaisante; pourtant, je lui trouve un caractère poignant, mystérieux et plutôt mélancolique ${ }^{11}$. »

Lorsque l'on écoute l'enregistrement de l'onde carrée produite par la saqueboute électronique, on entend une forme d'onde simple avec des changements variés et subtils de hauteur et de volume, et dont le timbre est remarquablement semblable à celui de la clarinette.

Le Caine avait construit un instrument qui établissait de manière convaincante ses théories sur la nature du son musical. Son raisonnement était le suivant:

L'écoute attentive d'une voix musicale simple révèle que l'interprète, jouant d'un instrument monophonique classique (qui ne produit qu'une note à la fois) tel le violoncelle, le saxophone ou la voix humaine, dispose de la maîtrise continue et précise des trois paramètres musicaux: la hauteur du son, le volume et le timbre.

Alors que l'oreille est capable de reconnaitre plusieurs milliers de sons ayant des hauteurs différentes, moins d'une centaine d'entre eux peuvent être écrits sur une partition musicale, et l'élaboration des idées principales s'effectue dans un intervalle encore plus restreint. Notre système de notation musicale ne fournit qu'une indication sommaire des désirs du compositeur de s'écarter des sons choisis; les musiciens qui utilisent des instruments expressifs commettent régulièrement des entorses minimes, qui tiennent un grand rôle dans l'effet produit par un instrument monophonique. La continuité de la ligne sonore d'un instrument monophonique constitue une différence importante avec les instruments polyphoniques comme l'orgue. Certains types de souplesse tonale sont impossibles à rendre sur un instrument polyphonique de ce genre, dont la principale caractéristique est la séparation de chaque voix en sons discrets, produits par des générateurs séparés.

Ainsi, l'instrument monophonique est l'instrument de musique fondamental, et le point de départ de toute la pensée musicale; l'instrument polyphonique, comme l'orgue, est tout au plus un expédient, dont la nécessité s'explique par la difficulté de réunir le nombre requis d'instruments monophoniques et de musiciens ${ }^{12}$. 
La saqueboute électronique était un instrument monophonique destiné à être utilisé pour les concerts en direct. Le Caine l'avait conçue à l'origine comme un instrument dans lequel la pression sur les touches pouvait être exercée en trois dimensions, chacune correspondant à un paramètre de la sonorité musicale. La pression verticale devait agir sur l'intensité; la pression latérale de gauche à droite changeait la hauteur, et la pression latérale vers l'interprète, ou loin de lui, devait agir sur le timbre. Cette organisation avait pour but de rationaliser les commandes.

Le concept de timbre en tant que paramètre unidimensionnel fut rapidement abandonné. Trois dispositifs de commande distincts furent installés sur le haut de l'instrument, d'où ils étaient actionnés par la main gauche. Chacun d'entre eux permettait à l'interprète d'effectuer des changements graduels et subtils de sonorité tout en jouant. Une organisation plus simple bidimensionnelle fut employée pour le clavier, à partir duquel les changements de hauteur et d'intensité étaient effectués par la main droite. Les touches étaient sensibles à la pression des doigts verticalement et latéralement (de gauche à droite). Les variations de pression selon l'axe vertical changeaient le volume de la note. Il s'agissait là d'une caractéristique importante dont ne disposèrent pas les musiciens avant les années 1980. Le pianiste peut jouer doux ou fort, mais il ne contrôle pas la durée de la désinence. En revanche, le joueur de saqueboute électronique pouvait augmenter ou diminuer le volume de la note pendant que celle-ci résonnait. La hauteur du son était contrôlée au clavier, non seulement par les différentes notes, mais également en exerçant une force latérale plus ou moins grande sur la touche, ce qui produisait un glissando ou un vibrato. La technique du clavier ressemblait à cet égard à celle des ondes Martenot. [...] L'action des doigts servant à produire le vibrato était la même que chez les violonistes.

En 1945, Le Caine testa plusieurs types d'oscillateurs disponibles sur le marché afin d'en trouver un pour la saqueboute, et il conclut de ces essais que les oscillateurs commandés en tension étaient supérieurs. Il adopta également la commande en tension dans les amplificateurs et les filtres, et utilisa une variété de formes d'onde pour générer différents timbres. Cet ensemble de techniques fut plus tard connu sous le nom de synthèse sonore analogique. La saqueboute fut en l'occurrence le premier synthétiseur.

L'aspect extérieur de la première saqueboute ne reflétait aucunement ses caractéristiques musicales novatrices. Son support était constitué de planches de boîtes d'emballage sur lesquelles subsistaient encore quelques bouts de chiffon et quelques agrafes. Les planches de longueurs inégales étaient clouées sur les trois pieds de support à des angles différents. Le haut de 
l'instrument était une simple feuille de contreplaqué sur laquelle étaient écrites au stylo des indications sur les fonctions des commandes. En haut à droite était marqué l'avertissement suivant: «Attention : prière de laisser le tournevis sur l'instrument. » Le clavier était constitué de touches de bois apparemment sculptées à la main dont le haut était incurvé.

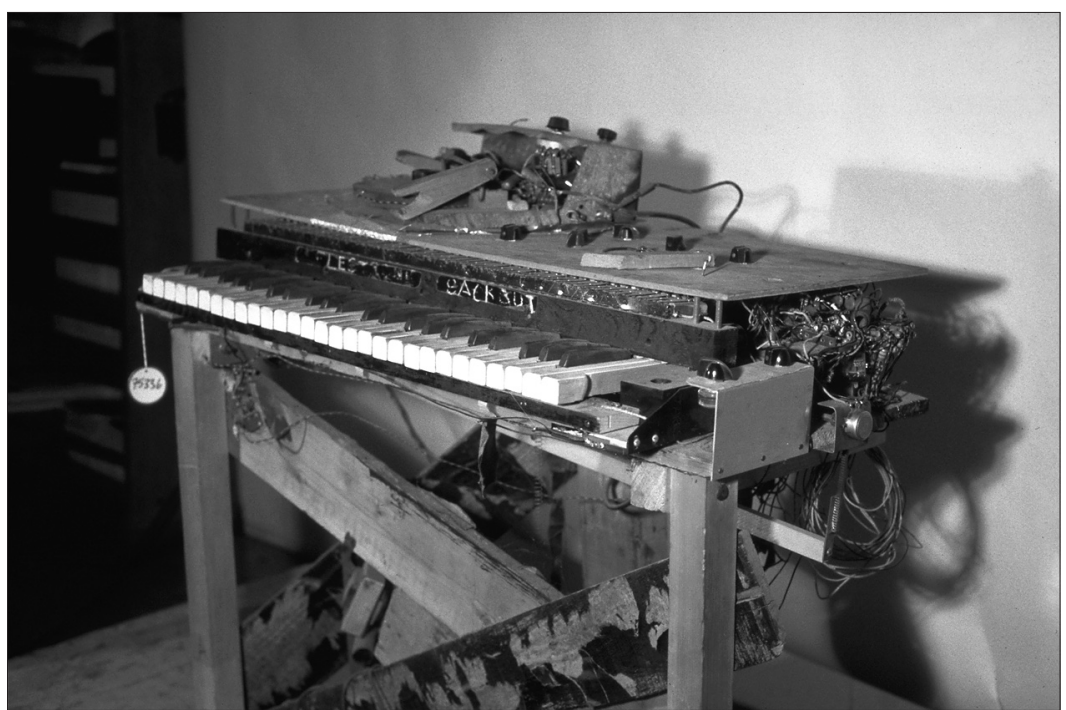

Prototype de la saqueboute électronique de Hugh Le Caine.

Photo : (c) Gayle Young. Reproduite avec la permission de l'auteure.

Le Caine avait construit la saqueboute lui-même dans son studio, à l'aide de méthodes semblables à celles qu'il employait au laboratoire du CNRC. Toutefois, au CNRC, il disposait de l'assistance technique de Gordon Ellis, avec lequel il travaillait depuis le projet du radar. Bill Keen, un de ses collaborateurs à la station de recherche, se souvient de la manière dont Ellis s'adaptait aux habitudes de travail de Le Caine:

Hugh avait l'habitude de travailler sur ses circuits en prenant un marteau et un clou et en enfonçant le clou dans une poutrelle... Il avait une balle de corde de boucher, et quand il attachait la corde au clou, la balle tombait par terre... Il s'attaquait alors au premier circuit qu'il voulait, et s'emparait d'une douille. Avant l'apparition des transistors, on disposait de tubes qui ressemblaient à de petites ampoules de verre avec des broches à l'extrémité, que l'on devait faire glisser dans une douille. Les premiers tubes étaient assez volumineux, d'un diamètre d'un pouce et demi et d'une longueur de quatre pouces. Les douilles étaient grosses, elles possédaient huit broches et étaient entourées d'une bride métallique percée de deux trous servant à les fixer au châssis métallique, Hugh prenait ces douilles et attachait la corde à 
et l'essayait à l'aide des oscilloscopes, et ainsi de suite jusqu'à ce qu'il produise ce qu'il désirait; il attachait ensuite le support suivant, faisait les connexions avec les pièces, les résistances et les condensateurs qui pendaient dans le vide. Imaginez un crayon très fin, coupé en petits morceaux d'une longueur d'un demi ou d'un pouce, avec des fils qui sortent des extrémités, et portant différentes bandes de couleur, c'est cela une résistance, et certains condensateurs étaient un peu plus grands, un demi-pouce de diamètre, un pouce et demi de long, avec des fils qui sortent des deux extrémités.

Hugh fixait ces pièces sur le support, le connectait à celui au-dessus et, s'il avait besoin de plus d'espace, se levait et faisait un nœud dans la corde pour la raccourcir. Pendant la nuit, il finissait son produit, qui pouvait être constitué de huit supports, avec tous les composants pendus dans tous les sens, et de petits fils d'environ un pied et demi, ou peut-être deux pieds de longueur. Il le décrochait du plafond et le descendait, le plaçait sur le banc et laissait une note disant: «Gordy, pourriez-vous fabriquer l'un de ceux-ci?»

Gordy devait le faire, et aller chercher les autres composants et les installer, le premier support d'abord, le deuxième ensuite, et les aligner de sorte qu'il n'y ait pas de feedback, et disposer les autres composants; il devait ensuite aller à l'atelier de fabrication de pièces métalliques et faire fabriquer une pièce de laiton ou la faire lui-même, la courber, la peindre, la câbler et la terminer seul si c'était possible. Il présentait alors à Hugh le produit fini.

Lorsque Hugh a construit la saqueboute électronique, il a fait plus ou moins la même chose. Les composants pendaient comme des spaghettis et il fallait les réinsérer. Vous pouvez imaginer le nombre de courts-circuits et de problèmes occasionnés par ce travail, mais cela ne le gênait pas. Si cela ne marchait pas, nous savions exactement quoi tirer et quoi pousser jusqu'à ce que cela marche ${ }^{13}$.

Le Caine pensait qu'au $x^{\mathrm{e}}$ siècle, le progrès en musique ne tendrait pas nécessairement vers le remplacement des vieilles idées par de nouvelles, mais plutôt vers l'élargissement des ressources du musicien. Il a mis à la disposition de l'interprète certains moyens d'expression qui lui étaient familiers, mais les possibilités nouvelles qu'offraient ses instruments allaient bien au-delà de celles des instruments traditionnels. Le Caine utilisait souvent la musique classique traditionnelle pour mettre à l'épreuve les ressources musicales de son instrument, mais il pressentait que celles-ci se situaient au-delà d'une approche strictement classique.

Certaines techniques employées dans la saqueboute témoignent du souci de Le Caine d'adapter la technologie aux besoins du musicien. Les oscillateurs à tubes à vide avaient été utilisés pour la production sonore pendant quelque temps avant 1945, mais leur utilité pour les instruments de musique était limitée du fait que leur fréquence variait linéairement avec la tension. La gamme musicale requiert une augmentation logarithmique de la
13. Entrevue, William Keen. La saqueboute de 1947 est maintenant au Musée national des sciences et de la technologie. 
14. Les plans relatifs au dossier des brevets font partie du fonds Le Caine, à la Bibliothèque nationale du Canada. Le Caine avait déjà commencé à distinguer les méthodes de commande analogique et numérique. fréquence, les différences de fréquence entre les notes s'accroissant à mesure que la hauteur s'élève. La solution ingénieuse de Le Caine à ce problème fut de connecter les touches du clavier à l'oscillateur par l'intermédiaire d'un fil à résistance, qui modifiait la tension de manière proportionnelle à sa longueur. Chaque touche était reliée par une prise en un point différent du fil à résistance, et envoyait une tension différente à l'oscillateur, fournissant ainsi la fréquence exacte pour chaque note.

Tout en travaillant à la conception de la saqueboute, Le Caine se constituait un dossier contenant les brevets déposés sur les instruments électroniques. Il avait divisé ces brevets en trois catégories, correspondant aux trois aspects de la conception des instruments: les circuits, l'organisation et les commandes. Les circuits, qui constituaient l'aspect technique de la production sonore, incluaient les rouages du son et les oscillateurs à tubes à vide. L'organisation avait trait à la façon de jouer l'instrument. Les commandes pouvaient être de simples interrupteurs on-off, ou encore des dispositifs sensibles au toucher, photosensibles ou sensibles au mouvement, qui servaient à modifier graduellement la sonorité. Il est intéressant de noter que, pour Le Caine, ces trois aspects de la conception des instruments revêtaient une même importance. Nombre d'inventeurs se concentraient sur un seul d'entre eux, sans référence aux deux autres. Le brevet sur l'orgue Hammond, nota Le Caine, était l'un des seuls qui décrivait les trois aspects de l'instrument. Le Caine avait conçu un système complexe de classement des brevets sur les instruments avec des renvois aux trois aspects de la conception et un fichier central répertoriant chaque brevet par numéro ${ }^{14}$.

À l'été de 1946, les travaux sur la saqueboute avaient suffisamment avancé pour que l'on puisse en jouer comme d'un instrument à clavier, et l'instrument fut essayé non seulement par Le Caine mais par plusieurs de ses amis. Le Caine organisait régulièrement chez lui des séances de musique improvisée, dont certaines étaient enregistrées sur des disques d'acétate. Sur l'étiquette de l'un des enregistrements du premier été, on peut lire l'indication suivante : «Bill Farrow joue de la saqueboute - 194615.» D'autres améliorations furent apportées à l'instrument. En 1945, Le Caine «ajouta un roulement à billes au mécanisme de l'analyseur de mouvement, celui qui établit la distinction entre les changements de fréquence et les changements d'intensité [sur le clavier]. Ce dispositif facilite un peu le jeu. Aucun changement de hauteur ne se produit à présent lorsqu'une note est attaquée violemment ${ }^{16}$. » En 1947, il apporta encore d'autres raffinements sonores:

Je travaille à une méthode par laquelle de légers changements de hauteur du son peuvent altérer le timbre de manière appréciable, et ce, toujours pour la même rai- 
son, pour éviter d'obtenir un son mécanique. Je travaille également à un circuit qui transforme la transition entre deux notes en un changement graduel de hauteur. La constante de temps peut être si longue que le son ressemble à une liaison exagérée, ou si courte que l'on a alors l'impression d'un changement abrupt ${ }^{17}$. [...]

\section{Compositions de musique électronique}

Le Caine, habitué à travailler dans l'isolement, décida d'inventer des instruments qui feraient de la musique nouvelle une réalité. Après avoir destiné ses premiers instruments à l'interprétation de musique traditionnelle, il expérimenta de nouvelles techniques et étudia en détail la musique du XX⿳亠丷厂犬⿱ commencer par Busoni et les futuristes:

Les futuristes, au début du siècle, ont vu la nécessité d'une nouvelle musique qui serait l'expression du monde nouveau et des mutations sociales qui virent le jour à mesure que l'industrialisation et la technologie constituaient une nouvelle base pour la vie et réorientaient les forces vitales. Ils ont formulé une esthétique qui puisse utiliser les sons, les signes, les odeurs et le climat social de la première décennie de ce siècle à la place des sons et des visions pastorales du temps de Beethoven ${ }^{18}$.

Le Caine avait remarqué que, de tous les nouveaux instruments du XXe siècle, aucun ne permettait au compositeur de se soustraire aux limites de l'orchestre traditionnel, même si par les sons produits et la façon d'en jouer, ils différaient par rapport aux instruments classiques. Il est en effet surprenant de constater à quel point la musique a été dominée par la nature des instruments classiques, même lorsque ceux-ci n'apparaissaient pas sur la partition ${ }^{19}$.

Le Caine remarquait que les œuvres les plus novatrices n'étaient plus composées pour des interprètes, mais pour ce qu'il appelait des «systèmes de stockage et de lecture». Le terme de «musique électronique » référait davantage à cette tendance qu'aux instruments électroniques de concert plus anciens : «L'aspect essentiel dans la technique de la musique électronique est que l'on ne met maintenant plus l'accent sur le son en direct qui existe dans le temps, mais sur le son enregistré qui est intemporel ${ }^{20}$. "

Pierre Henry fut l'un des premiers compositeurs à faire usage de sons enregistrés sur un phonographe, appareil que Le Caine décrivait comme «un dispositif très répandu qui a connu pendant environ soixante-quinze ans une vie relativement calme et respectable, et qui, soudain, a révolutionné les perspectives des compositeurs ${ }^{21} »$. Des années plus tard, Le Caine a évoqué l'influence décisive qu'eut sur lui Antiphonie, œuvre composée par Henry en 1952 :

La pièce qui, à mon sens, révéla le monde musical radicalement neuf que nous connaissons maintenant fut Antiphonie de Pierre Henry... À cette époque, je
17. Le Caine à Bill Farrow, novembre 1946. Le Caine fit des lectures approfondies en psychoacoustique, et fit référence en particulier aux cinq volumes de C. Seashore intitulés The Psychology of Music, New York, McGraw Hill, 1938.

18. Le Caine, "Recherches au temps perdu ", Op. cit., p. 59.

19. Le Caine, "Electronic Music Today", (inédit, 1966).

20. Ibid.

21. Le Caine, "Electronic Music in 1954", (inédit, 1959). 
22. Entrevue avec Le Caine par Alan Gillmor et William Wallace, 11 octobre 1975 .

23. Le Caine, "The Electronic Music considérais la Suite lyrique de Berg comme le summum de la difficulté et de la provocation intellectuelle qu'une pièce pouvait atteindre sans devenir absurde ou dénuée de sens [...] Antiphonie me semblait briser toutes les règles musicales afin de provoquer en même temps du dégoût et une vive curiosité [...] C'est une œuvre qui m’a hanté pendant des années: la juxtaposition délibérée d'effets discordants organisés sous une forme très rigoureuse. Ce qui m’impressionne est qu'ils puissent être structurés de cette manière. C'est la rupture avec la fluidité de l'écriture pour orchestre classique ${ }^{22}$.

La musique produite par les techniques d'enregistrement sur bande se développait parallèlement à la musique instrumentale de la même période. Les compositeurs faisaient de plus en plus usage de structures rythmiques non métriques et de méthodes d'organisation sérielle. De plus, les limites de ce qui pourrait être appelé le «son musical» reculaient. Toutefois, pour Le Caine, c'est la musique électronique qui véhiculait l'excitation de la découverte. Cette musique sortait de la relation temporelle normale, de l'échelle temporelle de l'interprétation en direct, et explorait l'effet hypnotique de la répétition simple.

L'enregistrement sur bande magnétique rendait possible la manipulation et le stockage du son. Pierre Henry et Pierre Schaeffer, dont l'Étude pour moulins à vent construite à partir de sons enregistrés sur disque fascinait Le Caine, commençaient à élaborer une banque d'objets sonores allant des enregistrements effectués sur une voie ferrée à ceux d'une pièce de monnaie rebondissant sur une table. Au début des années 1950, le phonogène fut construit pour le Studio de musique concrète de Paris. Cet instrument permettait de faire passer une boucle de bande à douze vitesses de lecture différentes correspondant aux douze notes de la gamme tempérée. Il permettait également de faire varier la vitesse en continu et de produire des changements progressifs. La manipulation des bandes magnétiques était une technique nouvelle. Les sons pouvaient être fixés sur bande, puis être joués et rejoués, évalués et modifiés. Dans une de ses conférences, Le Caine souligna que, avant l'avènement de ces techniques, les sons du monde environnant ne pouvaient pas être utilisés en musique car on «ne pouvait pas leur apprendre à jouer». Un grand nombre de compositeurs en Europe et en Amérique du Nord étaient fascinés par l'idée d'assembler des sons naturels pour former de nouvelles séquences que l'on ne pouvait obtenir par aucun autre moyen²3.

Des années plus tard, Le Caine s'est rappelé sa première expérience de manipulation de bande magnétique:

Lorsque j'étais en Angleterre, j'avais écouté au Third Programme de la BBC (en 1950 ou 1951) une émission sur Pierre Schaeffer. Là-bas, je ne disposais ni de magnétophone ni de studio, et j'ai dû attendre d'être revenu pour essayer ce procédé, ce que 
j’ai fait dès mon retour. Tout son nouveau était alors enthousiasmant. On enregistrait le bruit d'une bouteille qu'on cassait ou le son de balles de ping-pong - j'ai utilisé cet effet pendant longtemps. On pourrait croire qu'il s'agit d'un son très monotone et dépourvu d'intérêt mais, à cette époque, tous les sons étaient passionnants. On approchait le son avec une perception nouvelle, car le simple fait de répéter un son constituait une expérience. Deux gouttes d'eau ne rendent jamais le même son dans la nature. Être en mesure d'isoler même un simple son et de le jouer deux fois était une expérience, et on pouvait l'écouter aussi longtemps qu'on le désirait, puis l'ajouter à sa connaissance des possibilités [...] en termes de ce que l'on pouvait faire avec $[\ldots]$ je me rappelle avoir fait 500 collages en une fin de semaine [...] c'étaient les beaux jours de la musique concrète ${ }^{24}$.

De retour à Ottawa, loin du Third Programme de la BBC, il fut difficile pour Le Caine de se tenir au courant des nouvelles compositions. Il était presque totalement tributaire des émissions de radio et, malheureusement, la station de la CBC à Ottawa ne diffusait pas la nouvelle émission Music in Our Time. Le Caine devait écouter les émissions provenant de Montréal ou de Toronto, mais la réception était mauvaise. À l'été de 1955, il écrivit à la CBC à Toronto afin de demander des renseignements sur la disponibilité des enregistrements qu'il avait récemment entendus à la radio. John Beckwith, le producteur et présentateur de l'émission, lui envoya de la documentation sur les enregistrements publiés ainsi que des exemplaires de périodiques musicaux récents. Toutefois, Le Caine ne put emprunter les bandes de l'émission. Il commanda les enregistrements disponibles et continua à écouter Music in Our Time en dépit des bruits et des parasites ${ }^{25}$.

Beckwith organisa une émission spéciale sur la musique concrète à l'automne de 1955. Peu de temps après sa diffusion, Le Caine entreprit de perfectionner son multipiste de $195^{2}$ afin d'être en mesure de modifier les vitesses de lecture. Le nouvel appareil était destiné à faciliter la composition de musique concrète:

J'étais maintenant en mesure d'élargir et d'affiner les possibilités du multipiste. Je pus obtenir par l'intermédiaire du CNRC des magnétophones à bande destinés aux professionnels (Ampex), ce qui rendait le travail au studio beaucoup plus fonctionnel. J'ai progressivement changé de méthode en enregistrant sur l'Ampex et en faisant rejouer l'enregistrement sur le multipiste. Depuis que j’étais passé du disque d'acétate à la bande magnétique en 1953, j’étais frappé par les immenses possibilités qu'offrait la manipulation des bandes par rapport à celle des disques. Mes intérêts allaient de plus en plus de la musique traditionnelle à la musique concrète ${ }^{26}$.

Le premier problème qu'éprouva Le Caine pour affiner le multipiste fut de trouver un dispositif mécanique capable de contrôler la vitesse avec précision et de la changer rapidement. À cette époque, Lorne Bradley, un ami de
24. Entrevue avec Le Caine par Gillmor et Wallace, 11 octobre 1975 .

25. Le Caine à John Beckwith, 24 août $1955 ; 20$ septembre 1955. John Beckwith à Le Caine, 22 août 1955; 31 octobre 1955; 3 novembre 1955.

26. Le Caine, "Recherches au temps perdu ", Op. cit., p. 53. 
27. Entrevue, Lorne Bradley. II semble qu'il se soit passé quelque temps avant que Bradley puisse récupérer l'appareil du laboratoire de Le Caine.
28. Le Caine, "Recherches au temps perdu ", Op. cit., p. 53-54. Un extrait sonore de Dripsody se trouve à l'adresse suivante: <http://www.hughlecaine. com/sounds/dripsody.mp3>.
Le Caine, utilisait un intégrateur de disque à bille pour ses travaux dans un autre laboratoire. Il s'agissait d'un disque tournant, doté en son centre d'une bille mobile. La position de la bille commandait la vitesse de rotation du disque et pouvait être changée au moyen d'un dispositif d'entrée. Le Caine pressentit immédiatement le potentiel de ce disque pour l'entraînement du multi-track; une fin de semaine, il l'emporta dans son atelier et commença à y travailler ${ }^{27}$.

Le prototype du multipiste pouvait jouer six bandes sur un seul galet d'entraînement sur la gauche de l'instrument, tandis que les changements de vitesse étaient commandés sur la partie droite. Cet appareil était en fait un lecteur de bandes multiples à vitesse variable, mais il conserva son nom informel de 1952. On l'appelle toujours «magnétophone multipiste» ou «multi-track».

Dès que le prototype fut opérationnel, Le Caine l'utilisa pour achever sa première composition :

Dès la fin de 1955, j'ai produit Dripsody ( «Rhapsodie d'une goutte d'eau») sur mon magnétophone multipiste à vitesse variable. J'avais travaillé pendant des mois à une composition intégrant trois objets sonores: le bris d'une vitre de verre par un marteau, le son d'une balle de ping-pong heurtant la raquette et le son de la chute d'une seule goutte d'eau. J'avais tenté plusieurs essais, mais aucun n'avait réussi. Une nuit, vers trois heures du matin, je me suis dit: "pourquoi ne pas essayer la goutte d'eau toute seule? » Dripsody était une expérience où j'utilisais une vitesse de lecture variable sur le son rendu par la chute d'une seule goutte d'eau enregistrée sur une portion de bande très courte ${ }^{28}$.

La pièce porte en sous-titre «Étude pour magnétophone à vitesse variable». On peut lire ensuite: «Cette œuvre est dédiée à Lorne Bradley, à qui je dois le système d'entraînement à vitesse variable. » Le Caine ajoute à l'œuvre les remarques suivantes:

On faisait tomber l'eau d'un compte-goutte maintenu à environ dix pieds d'une corbeille à papier métallique contenant deux pouces d'eau. Un microphone était placé dans la corbeille, isolé de l'eau par un couvercle de plastique. Comme il fallait un endroit très calme, on plaça le magnétophone dans le corridor, et on ferma les portes menant aux deux pièces. À partir d'une bande d'une demi-heure, on a choisi le son d'une seule goutte. Certains sons oscillants donnaient à la goutte un son gargouillant; d'autres glissaient sur plus d'une octave. Pour simplifier le problème de la composition, on a choisi le son le plus simple et le plus informe, mais c'est avec regret que l'on a éliminé les gouttes ayant des changements de hauteur complexes.

Un enregistrement du son choisi dont la longueur était d'environ un demi-pouce a été monté sur une boucle de bande, entouré bien entendu d'une bande non magnétique de sorte que les bruits non désirés ne soient pas reproduits. Les figures rythmiques, simulant les rythmes de l'eau qui tombe goutte à goutte, ont été écrites 
en notation musicale. Naturellement, j’ai choisi les figures qui me semblaient les plus caractéristiques. L'eau qui tombe goutte à goutte rend des sons qui varient énormément en hauteur, en durée, en rythme et en forme. La physique de la production des sons est obscure. Du fait de la brièveté des sons, on imagine souvent que l'eau tombe dans une certaine gamme ou un certain accord, mais il s'agit d'un effet psychologique. J'ai choisi la gamme pentatonique pour représenter l'ordre imposé sur le schéma tonal par l'auditeur. Je ne pense pas que les sons engendrés par des gouttes d'eau appartiennent à cette gamme, mais je crois que nous avons tendance à utiliser notre expérience auditive passée pour assimiler un nouvel ensemble de sons que nous écoutons, et à ajuster les cas limites de sorte qu'ils s'intègrent à une organisation prédéterminée. Bien que les premières parties de la pièce constituent une présentation relativement réaliste de la chute de gouttes d'eau, toutes les notes appartiennent à la gamme pentatonique. Le premier son que l'on entend est l'enregistrement initial non altéré.

Dans l'enregistrement écrit, on avait placé derrière chaque note un chiffre d'intensité en décibels lui correspondant. Les valeurs de temps des notes étaient transcrites en longueurs de bande au moyen d'une échelle en papier.

Pour l'élaboration de la matière musicale, toutes les opérations faciles à effectuer sur un magnétophone ont été utilisées. Dans cette courte composition, on retrouve 1000 impressions de l'enregistrement original sur bande du son de la goutte d'eau, même si un petit nombre seulement de montages fut effectué. Quatre boucles de bande ont été montées, dont la boucle originale sur laquelle était inscrit l'enregistrement du son de la goutte. Les sons brefs sur les trois autres boucles ont été transcrits à partir de rythmes trouvés dans l'enregistrement de la chute des gouttes, comme nous l'avons expliqué plus haut. En imprimant différentes combinaisons des quatre boucles à trois vitesses différentes en même temps, on a pu obtenir l'effet de douze boucles de bande sans devoir procéder à d'autres montages. Les trois vitesses étaient reliées par un facteur de deux, de sorte que les sons soient en rapport d'octave. Il est surprenant de constater le changement créé dans le caractère du motif par l'introduction d'un facteur de deux dans l'échelle de temps.

Dans la section d'arpèges, un seul arpège de douze notes a été monté. Il est répété à des hauteurs différant d'une octave. Ensuite, l'effet suivant comportait plusieurs arpèges, ascendants ou descendants, à des hauteurs et à des vitesses différentes, joués au même instant. L'effet obtenu ressemble à celui que l'on crée sur la harpe en déplaçant les doigts des deux mains en un mouvement circulaire et dans des directions opposées.

En n'effectuant que vingt-cinq montages et en utilisant des techniques d'enregistrement, on produit une bande finale dans laquelle on perçoit des milliers de sons indépendants ${ }^{29}$.

L'œuvre complète, dont la longueur est de une minute et vingt-six secondes, fut achevée à $7 \mathrm{~h} 30$ du matin, et Le Caine la joua à ceux qui arrivèrent tôt au laboratoire.
29. Le Caine, "Notes on Dripsody" (deux versions) et "Recherches au temps perdu", Op. cit., p. 55-56. 
30. Ussachevsky à Le Caine, 2 novembre 1956. Le Caine a présenté ses idées dans "From the Labs: A New Music", un article paru dans le Financial Post du 29 septembre 1956. On trouvait un article portant sur le Composer-tron d'Osmond Kendall dans le même journal.
Vers la fin de 1955, alors que Le Caine travaillait à la conception du multitrack, Gordon Ellis et Horace Aubrey préparèrent la saqueboute pour une tournée en Ontario. Une fois Dripsody achevée, Le Caine décida brusquement d'emporter le multi-track à sa tournée au lieu de la saqueboute. Le multi-track était devenu son premier instrument. Il le pensait plus important que la saqueboute ou que l'orgue sensible au toucher, mais continuait néanmoins à travailler sur les trois instruments.

La publication de l'article de Le Caine dans les Proceedings of the Institute of Radio Engineers au printemps de 1956 donna à ses travaux un auditoire international. Il reçut plusieurs lettres demandant des renseignements sur la saqueboute ainsi que sur la série d'articles traduits par le CNRC. Il reçut entre autres une lettre de Vladimir Ussachevsky, un compositeur de musique pour bande travaillant au studio de Columbia-Princeton à New York. Il exprimait son intérêt pour les traductions du CNRC ainsi que pour l'orgue sensible au toucher. Il faisait également l'éloge de l'article de Le Caine qui englobait tant le point de vue du musicien que les solutions techniques de l'ingénieur $3^{\circ}$.

Plus tard au cours de la même année, Ussachevsky vint visiter le laboratoire du CNRC. Il fut particulièrement impressionné par la saqueboute, et demanda si l'on pouvait lui en fournir une pour le studio de Columbia-Princeton. En cas d'impossibilité, il était prêt à utiliser l'instrument du CNRC pour enregistrer des sons qu'il affinerait ensuite et utiliserait dans une nouvelle œuvre sur bande. Le Caine, intrigué par cette demande, hésitait pourtant à offrir à Ussachevsky d'utiliser la saqueboute, car il ne la trouvait pas suffisamment au point. Il était néanmoins curieux de savoir quels sons et quelles ressources de l'instrument serviraient à la composition sur bande. Il invita Ussachevsky à revenir au CNRC en avril 1957. La saqueboute ne pouvait pas être prête avant cette date, car les améliorations qu'on y apportait étaient encore en cours. Malheureusement, Ussachevsky dut modifier ses plans de voyage, et il ne réalisa pas d'enregistrement avec la saqueboute dans une composition ultérieure.

En mai 1956, les politiques du CNRC furent débattues au Parlement. B. G. Ballard, qui était alors directeur de la division où travaillait Le Caine, fit un rapport au Comité de la recherche de la Chambre des communes sur les différents projets entrepris par le CNRC. La saqueboute et le projet de musique électronique firent l'objet de discussions enflammées, comme l'atteste l'Ottawa Journal sous le titre «La saqueboute imite tous les instruments, même la cornemuse» :

Un orchestre symphonique complet pourrait être constitué de saqueboutes. Jusqu'à présent, la saqueboute ne pouvait imiter qu'un instrument à la fois. M. Ballard a 
déclaré avoir entendu la saqueboute imiter la cornemuse, et les sons qu'elle rendait étaient tout à fait crédibles. Les trombones, les saxophones, les violons et les violons folkloriques sont des jeux d'enfant pour cet instrument électronique. Il a déclaré que les instruments de musique électronique telle la saqueboute permettent aux compositeurs de produire une musique qui ne pourrait être jouée par les instruments classiques. Ainsi, un nouveau domaine de plaisir musical vient de s'ouvrir à nous.

Alistair Stewart, CCF, Winnipeg-Nord, s'est demandé pourquoi le CNRC consacrait du temps à la musique électronique. Il avait entendu des orgues électroniques et a demandé: «Pourquoi devriez-vous nous infliger ce genre de chose?» M. Ballard a défendu la recherche électronique sur les sonorités musicales. «Il s’agit, entre autres, de l'une de nos activités les plus rentables », a-t-il déclaré. Bien sûr, ces instruments intéressent principalement les amateurs, mais ces derniers représentent une proportion appréciable de la population ${ }^{11} \ldots$

Des comptes rendus de la discussion furent télégraphiés partout au Canada, et des articles furent publiés, portant des titres du genre: «Pas la peine de travailler tes gammes, sœurette, joue plutôt de la saqueboute. » Horace Aubrey se souvient de l'intérêt suscité par les débats au Parlement: «Notre projet fit l'effet d'un coup de canon à la Chambre et, bien sûr, une vingtaine de députés vinrent essayer de comprendre ce que nous pouvions bien fabriquer. À leur arrivée, ils étaient très sceptiques, mais ils repartaient enthousiasmés. Pendant quelques années, nous n’avons pas eu de problème ${ }^{32}$. »

Le Caine continua à développer son langage musical et à utiliser ses instruments en dehors des formes de la musique classique traditionnelle. En 1956, il composa pour l'orgue sensible au toucher une nouvelle œuvre qui, dans son exploration des timbres et de la texture, allait bien au-delà de tout ce qu'il avait fait auparavant. La pièce s'intitulait Ninety-nine Generators en référence aux 99 générateurs de sons de l'orgue sensible au toucher. L'instrument est utilisé de manière non traditionnelle, comme s'il faisait partie des ressources sonores d'un studio de musique électronique. Le Caine ajouta une pédale de changement de hauteur qui, adaptée d'un dispositif utilisé au laboratoire de physique, faisait varier la hauteur de l'orgue entier à l'intérieur d'une octave33. Le vibrato et le glissando ne sont pas habituellement exécutés sur l'orgue à tuyaux. Dans cette composition, au contraire, les légers glissements et les longs glissandi d'accords plaqués deviennent des caractéristiques structurales marquantes. En jouant de l'instrument de cette manière, Le Caine devenait le précurseur du synthétiseur polyphonique. Aucun traitement sur bande magnétique n'est utilisé, mais la résonance de l'orgue fut accentuée par l'endroit où fut effectué l'enregistrement. Un petit édifice en forme de dôme, appelé radôme, avait été construit à l'extérieur du M-50 pour loger une antenne de radar. Le Caine, qui appréciait ses qualités acoustiques particulières, avait emporté l'orgue là-bas et y avait joué la pièce34.
31. Ottawa Journal, 24 mai 1956. Douglas Leiterman du Southam News Services a fait part de la nouvelle le 26 mai 1956.

32. Entrevue, Horace Aubrey, octobre 1980.

33. Le registre était commandé par un "diviseur par deux" qui appliquait une commande en tension à tous les oscillateurs en même temps (douze oscillateurs par octave). Du point de vue du concept, l'orgue sensible au toucher et plusieurs synthétiseurs polyphoniques de la fin des années 1970 sont très similaires; il s'agit d'instruments à clavier munis de commandes de timbre actionnées à l'aide de boutons-poussoirs, et produisant un son à la fois.

34. Le Caine, "Notes on Ninety-Nine Generators" et "Recherches au temps perdu ", Op. cit., p. 56. Un extrait de 99 Generators se trouve ici : <http://www. hughlecaine.com/sounds/9ggenerators. mp3>. 
35. Le Caine, "Notes on This Thing Called Key".
Vers la fin de 1956, le multi-track avait encore été amélioré. Un clavier fut ajouté au tableau de commande servant à changer la vitesse de lecture. En décembre de la même année, Le Caine composa une autre pièce à l'aide du multi-track. This Thing Called Key était une simple démonstration de la variation de la vitesse de la bande sur un fragment d'une pièce pour piano très connue. Le Caine enregistra au piano le morceau appelé Chopsticks:

Une pauvre petite œuvre sans défense est enregistrée sur une boucle de bande et est jouée et rejouée tandis que l'on apporte des variations en changeant la vitesse de lecture. La première fois, on laisse en grande partie la pièce dans la tonalité originale. La deuxième fois, on module dans une tonalité située un demi-ton plus bas que la tonalité originale. Ensuite, on s'aventure dans des tonalités de plus en plus éloignées 35 .

Les changements de vitesse affectent la hauteur apparente ainsi que le timbre du son original. Lorsque la hauteur change, la vitesse de la pièce change également. On entend clairement les limites supérieure et inférieure de ce processus. La pièce se termine par ce que l'on pourrait interpréter comme un cri de frustration. Il est assez simple de jouer en direct, avec le multipiste sur la scène et l'opérateur contrôlant la vitesse de lecture. Une boucle de bande

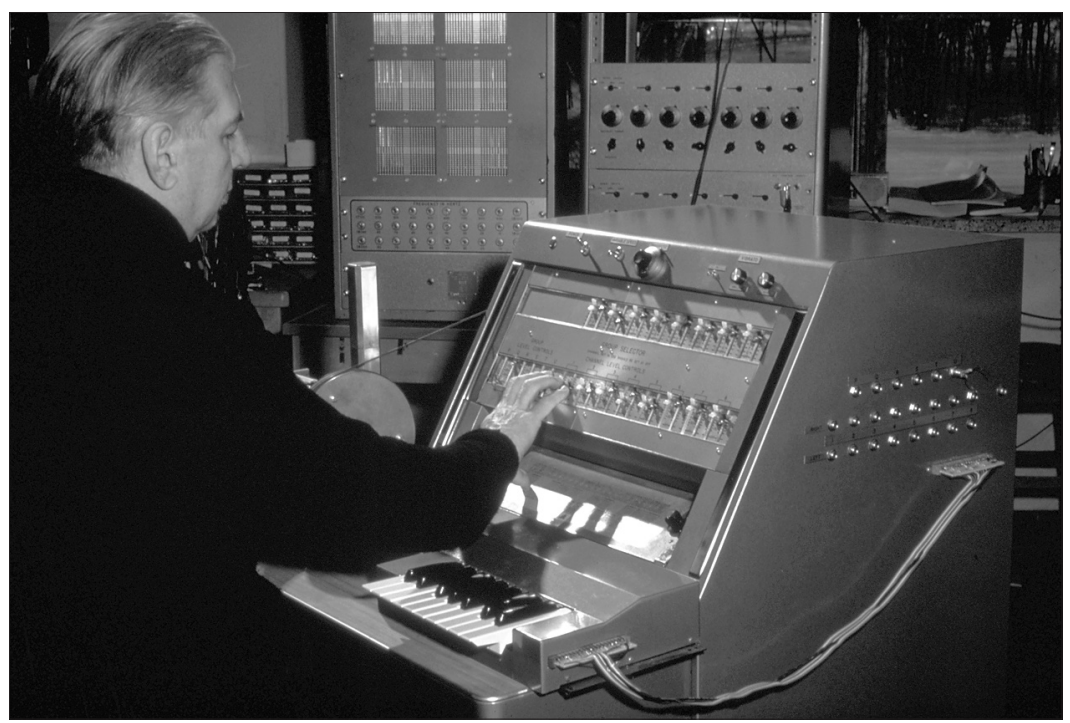

Hugh Le Caine utilise le dernier et le plus compact des cinq magnétophones à application spéciale au Conseil national de recherches du Canada, en 1967.

(c) Conseil national de recherches Canada. Reproduite avec la permission du ministre des Travaux publics et Services gouvernementaux Canada (2009) Source: Bibliothèque et Archives Canada/Fonds Hugh Le Caine. 
seulement est utilisée jusqu'au cri à la fin de la pièce. Cela aurait été un bon morceau de démonstration pour un virtuose du multi-track. [...]

Dans cette pièce, le penchant de Le Caine pour la farce est évident. Les deux émissions qu'il écoutait régulièrement à la radio étaient Music in Our Time et The Goon Show. Il était un grand admirateur de l'humour de Goon et enregistrait ses émissions ${ }^{36}$. Il affectionnait la juxtaposition de l'humour bouffon et d'effets sonores spéciaux. Son attitude face au son était espiègle, ce qui ne l'empêchait pas de travailler sur les effets musicaux expressifs qu'il voulait tirer de ses nouveaux instruments électroniques.

En dépit de l'aptitude admirable de Le Caine à organiser le travail de ses assistants et à monter de nouveaux projets, il éprouvait toujours une certaine difficulté à terminer un instrument, à déclarer que le prototype était satisfaisant et à passer au projet suivant. C'est pourquoi il avait d'ordinaire au moins trois instruments à la fois en cours de développement. Horace Aubrey comprenait que «Le Caine ne livrait aucun instrument avant qu'il ne soit absolument convaincu que c'était le meilleur du monde, et il n'en était jamais convaincu car il pouvait toujours l'améliorer37».

Le Caine avait travaillé pendant des années à affiner la saqueboute et l'orgue sensible au toucher, mais il restait persuadé que leur qualité sonore était insuffisante. En dépit des améliorations constantes qu'il apportait à ses instruments, il demeurait «un peu sceptique quant à savoir si l'être humain réussirait un jour à fabriquer des instruments électroniques aussi satisfaisants que ce qu'il avait fait avec les instruments à corde $3^{8} »$. Lorsque le fonctionnement d'un dispositif ne le satisfaisait pas totalement, il continuait à essayer de nouvelles techniques jusqu'à ce que le problème soit résolu. [...]

\section{Le multi-track}

À cette époque, Le Caine concentrait ses efforts sur l'amélioration du multitrack. Dès le début de 1957, l'instrument fut équipé d'un mélangeur élaboré et de sorties stéréophoniques. Quelques mois plus tard, il était doté du clavier de la saqueboute et du ruban de glissement qui servait à régler la vitesse de lecture. La tension de sortie du clavier était réglée de telle sorte que la vitesse des bandes doublait lorsque l'on enfonçait une touche une octave au-dessus de la touche précédente. La note sol au-dessus d'un do augmentait la vitesse d'un rapport de $3 / 2(1,5)$, soit l'intervalle d'une quinte juste.

Avec le multi-track doté de ces récentes améliorations, Le Caine décida de réaliser une deuxième version de Dripsody, cette fois en stéréo. La deuxième version était semblable à la première, mais elle était plus longue de presque une demi-minute, ayant une durée totale de près de deux minutes. La
36. Entrevue, Tony Johnson.

37. Entrevue, Horace Aubrey, octobre 1980. 
39. Le Caine à Carter Harman, Cook Laboratories, 26 février 1958.

40. Le Caine, "Notes on Dripsody" et "Recherches au temps perdu", Op. cit., p. 55. La technique décrite est semblable à celle qui a été utilisée dans Arcane Presents Lulu où elle permettait de modifier la hauteur d'un cri en glissando.

41. Le Caine, "Recherches au temps perdu ", Op. cit., p. 55 nouvelle version faisait davantage appel au traitement sonore et avait une texture plus complexe. Elle se terminait aussi plus abruptement. La première version, qui imitait le bruit de la pluie qui tombe, comprenait des périodes de silence dont la durée augmentait entre les derniers sons. Dans la deuxième version, on sait exactement quand le morceau se termine. Dans ses notes sur la deuxième version de Dripsody, Le Caine décrit ainsi les techniques qu'il a utilisées :

Dripsody illustre ce qui est arrivé la première fois que je me suis trouvé en possession d'un magnétophone à vitesse variable... juste avant les arpèges, j’ai introduit des gouttes fabriquées à partir de la goutte originale, mais ayant des glissements de hauteur semblables à ceux que j'avais observés dans les gouttes d'eau qui ne furent pas utilisées dans l'enregistrement de 1955. Pour les préparer, j’ai fait jouer le son de la goutte très lentement tout en faisant varier rapidement la vitesse. J'ai alors transposé les enregistrements obtenus dans le registre que je désirais. À ce sujet, je peux dire que ces sons sont beaucoup moins intéressants que ceux produits naturellement par les gouttes 39 .

Les sons glissants des gouttes sont entendus dans un «chœur spatial» dense, dans lequel on fait varier leur position apparente à l'aide de boucles de feedback multiples. Ces effets ont été réalisés à l'aide de rétroactions, de légers changements de hauteur ainsi que de gouttes aux formes variées. Deux sons légèrement différents étaient joués à peu près au même instant sur deux canaux, et créaient ainsi une impression générale d'espace et de mouvement. Dans le dernier accord formé par les sons des gouttes d'eau, trente-six boucles furent utilisées pour produire dix-huit sons, chacun allant d'un canal à l'autre à une vitesse différente. Tous ces effets furent créés sans utiliser la réverbération ${ }^{4}$.

Les changements de vitesse étaient produits sur le multipiste et enregistrés sur l'Ampex. Pour les changements de hauteur affectant l'ensemble de la masse sonore, Le Caine utilisa une autre technique :

Un procédé simple que l'auditeur n'entend jamais est l'actionnement du commutateur de vitesse durant la section des arpèges sur l'Ampex qui a servi à l'enregistrement final. Comme l'Ampex change de vitesse lentement par rapport au taux de répétition des notes, et comme les répétitions sont superposées en différentes couches, le glissement d'une octave est entendu comme un déplacement continu et comme un changement d'accord, les notes étant réarrangées par l'auditeur. En fait, il s'agit simplement d'un simple glissement d'une octave entière. La vitesse change en même temps, graduellement, par un facteur de deux. Si l'on avait utilisé des sons simples tenus, on aurait immédiatement reconnu l'effet comme étant un glissement d'une octave entière, dans le style de la guitare hawaïennet'.

Les sons de Rhapsodie d'une goutte d'eau varient entre 40 et 8 ooo Hz, et leur hauteur est nettement perceptible dans la majeure partie de cet intervalle.

[...] Depuis le début des années 1950, Le Caine avait énormément travaillé sur les mélanges de sons sinusoïdaux. L'incitation lui venait de Studie 
II de Karlheinz Stockhausen, qui utilisait des sons sinusoïdaux simples joués selon une structure rigoureusement ordonnée. Pour Stockhausen, les timbres et les textures de la pièce devaient être engendrés par les mélanges de sons sinusoïdaux plutôt que par les caractéristiques préétablies que l'on retrouve dans les instruments d'orchestre. Le Caine était impressionné par la technique de Stockhausen. Il affirmait que les mélanges de sons sinusoïdaux constituaient «les premiers sons réellement nouveaux rendus possibles par les techniques électroniques ${ }^{2}{ }^{2}$. Cette façon de parvenir à la maîtrise du timbre était appelée synthèse additive. Les sons sinusoïdaux étaient considérés comme les modules élémentaires à partir desquels on pouvait produire des sons complexes. En 1958, Le Caine avait conçu et construit un banc de 108 oscillateurs. Il s'agissait de douze rangées de neuf oscillateurs, chacun disposant de commandes séparées pour le volume, la hauteur et la forme d'onde. Toutefois, le contrôle du volumineux banc était difficile. Au début, Le Caine utilisait un clavier sensible au toucher. Chaque touche était connectée à l'oscillateur correspondant à un son donné, et le compositeur contrôlait le volume et l'enveloppe par la pression des doigts. Cette méthode était utilisée pour un petit banc d'oscillateurs au studio de l'Université de Toronto, mais elle était limitée par le nombre de contrôleurs que le compositeur pouvait manier simultanément. Afin d'assurer un meilleur contrôle, Le Caine décida de reconcevoir certains aspects du système de musique codifiée. Au début de 1959, il remonta le vieil appareil du sous-sol et se mit au travail. Il conserva la technique du papier à graphiques mobile pour introduire l'information dans la machine, mais utilisa cette fois des interrupteurs photosensibles. Le nouvel instrument fut appelé le spectrogramme. Une rangée d'une centaine de cellules photoélectriques lisaient l'information contenue sur le papier à graphiques d'une largeur de dix pouces, qui était marqué à l'encre aux endroits où le son devait être activé. Lorsque les parties sombres passaient devant les cellules photoélectriques, le nombre approprié d’oscillateurs généraient les sons.

En octobre 1959, Le Caine avait déjà synthétisé plusieurs chants d'oiseaux à l'aide du nouvel appareil, et il les fit entendre lors d'une conférence de l'Audio Engineering Society. Il était désireux d'étudier attentivement les chants d'oiseaux du fait de leur similitude avec les sons électroniques: «Les oiseaux, avec leurs organes mobiles très légers et leur important rapport force-inertie, peuvent produire une rapide modulation de fréquence, une rapide modulation d'amplitude et un rapide changement de ton ${ }^{43}$. »

Le spectrogramme offrait une méthode pratique de construire des sons électroniques complexes. On pouvait obtenir des sons glissants en changeant leur hauteur par faibles degrés. Toutefois, il était difficile de produire des sons bruyants ainsi que des sons de choc sur un support électronique. Pour
42. Le Caine, "Electronic Music in 1959 " (inédit). Ces notes indiquent que l'oscillateur était en mesure de produire des formes d'onde sinusoïdales, carrées, d'impulsions et en dents de scie dès 1958. Le Caine fit remarquer que les instruments électroniques antérieurs tentaient d'imiter les instruments acoustiques.

43. Ibid.; Le Caine à Joseph Tal, 29 janvier 1959. 
44. Extrait d'une entrevue avec Hugh Le Caine par Alan Gillmor et William Wallace, 11 octobre 1975. ces types de sonorités, le microphone et le magnétophone donnaient de meilleurs résultats.

Le Caine essayait de poser les bases d'un nouveau langage musical qui remplacerait le système mélodique et harmonique du XIX siècle. Il imaginait que le nouveau système serait constitué de sons «intelligibles» organisés en de nouvelles structures formelles, mais n’était satisfait par aucune de celles qu'il entendait. Les mélanges de sons semblaient être une orientation intéressante, mais il était impossible de prévoir ce qu'ils donneraient lorsqu'ils seraient transposés ou combinés l'un avec l'autre. Il était perplexe en constatant qu'un mélange de sons liés par une relation intégrale est perçu comme un son simple alors que, si le son fondamental ou le plus bas est laissé de côté, aucun effet n'est audible, à part un léger changement de timbre. La hauteur ne change absolument pas. Il doutait d'une preuve que l'oreille puisse percevoir les clusters sinusoïdaux selon des séries harmoniques et, par conséquent, doutait de l'intérêt d'une telle structuration. Le Caine avait l'impression que le fossé s'élargissait entre les principes de la construction sonore et son résultat perçu par l'auditeur, et concluait: «Certaines compositions de notre époque sont plus intéressantes à décrire qu'à entendre ${ }^{44}$.»

Il cessa de travailler sur le spectrogramme à l'automne de 1959 et consacra tous ses efforts à la reconception du multi-track. Un deuxième prototype du multi-track fut opérationnel dès la fin de l'été, après que le premier eut été envoyé à l'Université de Toronto. Un troisième instrument devait être construit pour le nouveau studio de Josef Tal à Jérusalem. Le Caine avait tout d'abord estimé qu'il serait prêt vers la fin de 1959. Mais ce n'est qu'un an et demi plus tard qu'il fut achevé. Le Caine avait décidé que l'instrument devait être de construction particulière, puisqu'il devait être envoyé à un studio situé à des milliers de milles du CNRC où sa réparation serait difficile. Il conçut un nouveau système d'entraînement des bandes afin d'en accroître la fiabilité, élargit les capacités de l'appareil afin de permettre la lecture simultanée de dix bandes stéréo, et incorpora quelques améliorations suggérées par les compositeurs de l'Université de Toronto.

Le premier multi-track avait été construit avec des tubes à vide; chacun d'eux possédait un élément chauffant. Les transistors étaient apparus sur le marché vers 1957. Ils ne comportaient pas d'élément chauffant, ne fonctionnaient que sur une tension de quelques volts et, ne requérant pas d'espace sous vide, étaient beaucoup moins fragiles. Dès leur apparition sur le marché, Le Caine les avait soumis à de nombreux essais. Il continua encore à utiliser les tubes pendant quelques années, mais utilisa des transistors lorsqu'il conçut le nouveau modèle du multipiste. Les transistors réduisaient le bruit produit par l'instrument et augmentaient sa fiabilité et sa stabilité. Ils 
étaient moins sensibles aux variations de température, étaient plus compacts et moins lourds. Le multi-track envoyé en Israël utilisait des amplificateurs et des préamplificateurs transistorisés mais, à cause du temps requis pour reconcevoir les circuits, les autres composants furent dotés de tubes à vide45.

Pendant ce temps, les travaux continuaient au studio de l'Université de Toronto. Myron Schaeffer avait été nommé directeur et assumait la responsabilité technique et pratique du fonctionnement du studio. Avec l'aide de Le Caine, il s'occupait lui-même de la maintenance de tous les appareils. Vers la fin de 1959, un technicien fut engagé pour le remplacer dans ces tâches, et Schaeffer put se consacrer à sa recherche et commencer à composer. À la fin de l'année, il avait composé trois nouvelles pièces au studio. Le Caine décrit les progrès des compositeurs dans une lettre à Josef Tal:

Ils commencent vraiment à travailler sérieusement, après avoir traversé une période de découvertes enthousiasmantes suivies d'amères déceptions causées par les limites des instruments électroniques. Par exemple, ils ont été fort déçus de ce que pouvaient faire les filtres électriques, car ils s'attendaient à ce qu'ils transforment le son de manière beaucoup plus spectaculaire ${ }^{4}$.

En janvier 1960, Schaeffer écrivit à Le Caine pour lui annoncer que les trois compositeurs du studio commençaient finalement à s'accoutumer aux techniques spéciales requises. Ils apprenaient à utiliser les instruments qui se trouvaient là, obtenaient des enregistrements plus nets et « des effets qui, même il y a trois mois, étaient encore un mystère47 ». Des années plus tard, Harvey Olnick remarqua qu'ils n'utilisaient peut-être pas le multipiste de la manière prévue par Le Caine. Ils avaient toujours des problèmes, car ils enregistraient d'ordinaire les sons plusieurs fois pour chaque modification. Ils se retrouvaient avec des sons de troisième ou de quatrième génération dans leur enregistrement final et donc avec une accumulation audible de distorsions et de bruits de bande. Olnick savait que Le Caine, en composant Dripsody, avait conçu mentalement l'organisation de la pièce avant d'entamer véritablement le travail au magnétophone. Il avait d'abord préparé la matière et construit les six boucles de bande utilisant des vitesses de lecture différentes. Il avait ensuite réalisé la version finale en utilisant des sons de première et de deuxième génération ${ }^{4}$. Le Caine avait passé beaucoup de temps sur les techniques de montage avant de construire le multipiste. Il avait élaboré sa propre méthode pour le travail sur bande en studio, lequel exigeait d'apporter une grande attention aux détails sonores. Il annotait souvent complètement ses pièces, ayant recours aux valeurs classiques de hauteur et de rythme, indiquant sur la partition tous les niveaux de décibels et les autres traitements. Il était ainsi en mesure d'achever une composition en studio en relativement peu de temps, comme il le fit en une nuit pour Dripsody.
45. Un paiement symbolique de $3000 \$$ fut effectué par l'intermédiaire de I'UNESCO à titre d'aide financière du gouvernement canadien à l'État d'Israël, car c'était la seule façon pour le CNRC de transférer ses biens à l'extérieur du pays.

46. Le Caine à Joseph Tal, 14 décembre 1959 .

47. Myron Schaeffer à Le Caine, 28 janvier 1960.

48. Entrevue, Harvey Olnick. 
49. D’autres studios des années 1950 sont décrits par David Keane dans "The Birth of Electronic Music in Canada", Studies in Music from the University of Western Ontario, vol. 9 (1984), p. 55-78.
50. John Bowsher à Le Caine, 26 octobre 1960; Myron Schaeffer à Le Caine, 13 décembre 1960. La composition de Myron Schaeffer Project $A$ fut la première pièce à associer l'hamographe et l'enregistreur multipiste. Le Caine avait déja utilisé des interrupteurs photoélectriques avec succès.
Le Caine n'avait nullement l'intention de montrer aux trois compositeurs de l'Université de Toronto comment utiliser le multi-track. Il était conscient que les limites de l'instrument lui-même déterminaient l'étendue de ses ressources, et ne voulait pas mettre davantage en évidence ses contraintes stylistiques intrinsèques en déconseillant le réenregistrement des sons. Il voulait que l'instrument s'adapte aux besoins des compositeurs et qu'il leur offre toute la souplesse voulue pour leurs expérimentations sonores. Il voulait que les compositeurs découvrent leurs propres méthodes.

L'université possédait l'un des studios de musique électronique les mieux équipés du monde ${ }^{49}$. Le Caine croyait qu'en tant qu'organisme public, le CNRC devait s'assurer la collaboration du studio pour la conception d'instruments standardisés susceptibles d'être fabriqués par des compagnies canadiennes. Il commença donc avec Schaeffer à élaborer le concept de studio modulaire, concept qui permettait à un studio de n'acquérir au départ qu'un petit nombre d'appareils, et de l'accroître progressivement. Par exemple, un appareil de lecture à quatre pistes et à vitesse variable pouvait plus tard être combiné avec la console de mixage et d'autres appareils de lecture, à mesure que le studio se développerait. La conception de studio modulaire mettait également l'accent sur les rapports entre les composants du studio. Le clavier du multi-track pouvait servir à programmer n'importe quel dispositif commandé en tension. Inversement, le mécanisme de lecture du multi-track pouvait être entraîné par n'importe quel dispositif engendrant une tension.

Myron Schaeffer commença à travailler sur un nouvel instrument qu'il appela l'hamographe, d'après les initiales des trois membres de la faculté qui travaillaient au studio: Harvey, Arnold, Myron. L'appareil utilisait six longueurs de film transparent d'une largeur de $35 \mathrm{~mm}$, sur lequel quelques surfaces étaient masquées par du ruban noir. Les bandes de film défilaient devant un interrupteur photosensible. Les zones noires du film réduisaient la tension à zéro lorsqu'elles passaient devant l'interrupteur, tandis que les zones transparentes augmentaient la tension au maximum. Les gradients étaient proportionnels à la surface du film couverte par le ruban noir. Les tensions servaient à contrôler le volume. L'instrument pouvait contrôler le volume de n'importe quel générateur, mais il était le plus souvent relié à la sortie à six canaux du multipiste. Il permettait d'obtenir un contrôle plus précis, dans les six canaux sonores, des attaques et des extinctions des enveloppes complexes, par rapport au contrôle avec six touches sensibles actionnées en temps réel. Il était ainsi possible d'obtenir des structures rythmiques complexes sans avoir recours au montage. L'instrument était particulièrement utile lors de la lecture de boucles de bande ayant des niveaux sonores continus ${ }^{\circ}$. 
En 1960, le multi-track connut une grande popularité. Deux studios américains voulaient l'acheter, un appareil devait bientôt être envoyé à Jérusalem, et le studio de la BBC en Grande-Bretagne était également intéressé. Une compagnie de Toronto, Electronic Associates Limited, avait fait part de son intérêt pour l'aménagement du studio modulaire. Il semblait que les instruments de Le Caine pourraient être vendus à d'autres studios. Le studio de l'Université de Toronto ne pouvait pas offrir suffisamment de temps de studio aux compositeurs intéressés, et il devint manifeste que le studio modulaire suscitait un vif intérêt. Schaeffer et Le Caine continuèrent à y travailler.

Durant l'été de 1960, István Anhalt réalisa sa Composition électronique $n^{\circ} 3$ au laboratoire du CNRC, à l'aide du banc d'oscillateurs et du spectrogramme. L'administration du CNRC inclinait de moins en moins à financer les travaux des compositeurs, et on proposa que le Conseil des Arts, qui n'existait que depuis trois ans, soit responsable de cet aspect. Toutefois, Anhalt fut invité à continuer à travailler au studio du CNRC, à la condition qu'il participe également à d'autres activités du laboratoire.

À la fin de l'été de 1960, l'International Conference of Composers eut lieu à Stratford, en Ontario, et Louis Applebaum, l'un des organisateurs, incita les compositeurs de l'Université de Toronto à produire quelques compositions pour la conférence. Henk Badings, Luciano Berio et Josef Tal devaient y participer et y jouer leurs œuvres. À la fin de 1960, Schaeffer avait achevé quatre nouvelles compositions personnelles, ainsi que deux œuvres en collaboration avec Arnold Walter et Harvey Olnick.

Le Caine fut invité à prendre part à un débat sur la musique électronique. Il décrivit les travaux en cours au CNRC et à l'Université de Toronto, et traita ensuite de la théorie de l'information et des processus stochastiques en tant que concepts susceptibles de faciliter la compréhension de la musique. À propos de l'utilisation des techniques électroniques par les compositeurs, il souligna: «Il s'agit peut-être de l'éternel besoin des artistes d'utiliser les matériaux de leur époque, tout comme nos sculpteurs délaissent leur burin pour s'emparer du fer à souder, et comme nos peintres abandonnent le pinceau pour la bicyclette ${ }^{51}$. À la fin de son exposé, Le Caine joua quelques extraits de la Composition électronique $n^{\circ} 3$ d'Anhalt. Il présenta cette œuvre en décrivant l'utilisation faite par Anhalt des groupes d'ondes sinusoïdales:

Le professeur Anhalt utilise une série d'ondes sinusoïdales similaires à celles que rendent les instruments aux sonorités métalliques, tels les cymbales, les cloches et les gongs. Ces séries ont été montées sur l'appareil que je viens de décrire [le spectrogramme] et ont été développées de manière à maintenir une plasticité de la structure sonore et de produire une sonorité fluctuante, scintillante et jamais constante $^{52}$.
51. Ndlr : Le Caine fait référence à la Roue de bicyclette (1913) de Marcel Duchamp.

52. Le Caine, "Synthetic Means", dans The Modern Composer and His World: A Report from the International Conference of Composers, Held at the Stratford Festival, Stratford, Ontario, Canada, August 1960. John Beckwith et Udo Kasemets (éd.), Toronto, University of Toronto Press, 1961, p. 109-133. 
53. Entrevue, Harvey Olnick. Dès 1960, Olnick s'était retiré de toute participation active et Arnold Walter était occupé à des tâches administratives. Ils continuèrent tous deux à collaborer avec Schaeffer à la production d'une pièce par année et ce, jusqu'en 1963.

54. Le Caine à Lejaren A. Hiller, 27 février 1959. Les détails relatifs au multipiste proviennent du manuel d'instructions, septembre 1961.
Au printemps suivant, après presque deux ans d'activité, le studio de l'Université de Toronto quitta la maison de Division Street pour s'installer au sous-sol de l'édifice Edward Johnson. L'édifice fut inauguré le 1er avril 1962. Quelques semaines plus tard, le studio fit l'objet de l'émission The Nature of Things, une série scientifique de la télévision de CBC. On y fit la démonstration du multi-track et on y parla de la musique électronique. Dans ses nouveaux locaux, le studio de musique électronique de l'Université de Toronto fut connu sous le nom d'UTEMS; cet automne-là, Schaeffer donna pour la première fois des cours de musique électronique aux étudiants diplômés en composition53. [...]

Comme Schaeffer, Le Caine était intéressé par la fusion d'approches différentes au sein du studio modulaire, dans lequel le multi-track conservait néanmoins un rôle central: «Nous envisageons une diversité considérable de techniques de composition et, tout en conservant quelques réserves vis-à-vis de la musique concrète, je pense que la manipulation des bandes, sous une forme ou une autre, sera un élément important des ressources de musique électronique dans les années à venir ${ }^{54}$. Il exprima ses réserves vis-à-vis des sons préenregistrés dans les notes d'une conférence qu'il donna vers la fin des années cinquante:

En dépit des progrès considérables réalisés depuis 1934 dans les techniques d'enregistrement, plusieurs aspects s'opposent manifestement à leur utilisation dans ce qu'Eimert a appelé «la composition de la musique », c'est-à-dire les deux processus distincts de la conception de l'œuvre musicale et de la production de l'enregistrement final.

Lorsque l'enregistrement est utilisé durant la conception de l'œuvre, c'est-à-dire lorsque différents sons, accords, thèmes ou développements de thèmes complexes sont déjà enregistrés sur le support avant même que la composition n'ait pris forme dans l'esprit du compositeur, leur existence sous forme concrète tend à inhiber la réflexion du compositeur. Ils sont, en fait, trop utiles en ce qu'ils suggèrent les développements et les orientations possibles de l'œuvre. Ainsi, le développement du thème est traité de manière trop rigide, trop mécanique. L'aptitude d'un compositeur à mémoriser et à articuler mentalement toutes les matières musicales avec lesquelles il travaille semble alors moins importante que sa capacité d'invention. Encore une fois, un dispositif permettant les variations, telle par exemple la répétition du même passage à des vitesses de lecture différentes, est efficace mais trop restreint pour qu'on en fasse un usage étendu. La musique concrète peut être qualifiée d'art mécanique dont le problème, comme celui de la photographie, est qu'elle en fait trop pour l'artiste. Comme c'est le cas pour la photographie, si chaque détail doit être réglé de manière rigoureusement conforme au plan soigneusement prévu par l'artiste, la méthode perd de sa valeur, car il serait plus facile de peindre que d'arranger la photo. Toutefois, lorsque l'artiste n'a pas grand'chose à dire, la possibilité d'exercer un contrôle absolu sur un procédé mécanique peut être pour lui la solution idéale... 
Il y a un autre inconvénient à utiliser des sons complexes en tant que support musical brut plutôt que des sons sans forme et plus plastiques. Lorsque le son possède une forme propre, le compositeur est confronté au problème d'adapter la forme du son à celle de la composition. Il est beaucoup plus facile de produire un tableau satisfaisant en utilisant de la peinture, qui n'a pas de forme propre, que de faire un tableau à l'aide de coquillages ou de perles de verre55.

Malgré ses doutes quant à la valeur de la musique sur bande, Le Caine continua à travailler sur le multi-track et, finalement, au début de mai 1961, l'instrument qui devait être envoyé à Jérusalem fut achevé. Il fut envoyé à l'UTEMS pour y être mis à l'essai, fut montré lors d'une conférence de l'Institute of Radio Engineers et fut réexpédié à Ottawa pour y subir les dernières modifications ${ }^{5}$. Il fut envoyé à Jérusalem en juillet. Cet été-là, alors que le laboratoire était encore désorganisé, un autre multipiste fut construit et doté d'améliorations importantes. Il possédait une armoire métallique d'allure moderne et des curseurs portant des codes de couleur; il était complètement transistorisé et offrait deux vitesses de lecture différentes sur la même tête d'entraînement; de plus, il possédait des voyants lumineux de couleur indiquant le mode dans lequel fonctionnait l'appareil. Il fut envoyé à l'UTEMS afin que chaque studio dispose de son propre multipiste.

Au cours de l'été de 1961, István Anhalt réalisa sa Composition électronique $n^{\circ} 4$ dans le laboratoire du CNRC, avec l'assistance financière du Conseil des arts. Il utilisa des matériaux sonores qu'il avait créés sur le synthétiseur de RCA au studio de Columbia-Princeton à New York. Il avait apporté les bandes à Ottawa et les avait modifiées, avait ajouté des sonorités à l'aide du spectrogramme et réalisé le mixage final au laboratoire ${ }^{57}$. Après son troisième été de travail au CNRC, Anhalt fit quelques suggestions quant à l'amélioration du studio. Il proposa l'acquisition de modulateurs en anneau et de filtres, et était enthousiasmé par la possibilité d'utiliser le spectrogramme comme élément du studio modulaire pour le contrôle des niveaux de bruit sur le multipiste.

Depuis 1960, toutes les demandes de multipiste avaient été transmises à la compagnie Electronic Associates de Toronto, qui envisageait de le fabriquer en tant que composant du studio modulaire. En juin 1962, la compagnie avait reçu de nombreuses demandes relatives à l'instrument, mais n’avait toujours pas obtenu les plans définitifs du CNRC. Elle écrivit à Le Caine pour lui demander les plans mais, en 1963, il était encore en train de modifier la conception et n'avait toujours pas soumis la version définitive ${ }^{8}$. Le projet de fabrication fut abandonné peu de temps après car on pensait qu'au moment où le prototype serait prêt, le marché aurait changé. Était-ce simplement que les compositeurs s'intéressaient davantage aux modulateurs
55. Le Caine, "Musical Sounds" (inédit, 1957).

56. Le Caine à István Anhalt, 27 avril 1961. La démonstration auprès de I'Institute of Radio Engineers eut lieu le 19 mai 1961.

57. Entrevue, István Anhalt, octobre 1980 ; Le Caine, "Recherches au temps perdu ", Op. cit., p. 61.

58. Geoffrey Miller à Vladimir Ussachevsky, 19 avril 196o. G. Miller envoya les plans du multipiste du studio modulaire à Ussachevsky. Il voulait l'aider à mettre au point un appareil en attendant que le multipiste soit vendu sur le marché Robert Erickson, du San Francisco Conservatory, dans une lettre à Le Caine datée du 21 août 1961, demanda des informations concernant l'achat d'un multipiste. Il écrit: "Cela semble être la solution à nos rêves." Demande de dessins et de plans définitifs par Electronics Associates de Toronto à Le Caine, le 12 juin 1962 (dossiers de correspondance du CNRC) 
59. Le Caine, "Recherches au temps perdu ", Op. cit., p. 65 de fréquence, aux filtres et à la génération électronique des sons? Il est plus probable qu'après un délai de trois ans, on pensait que l'instrument ne serait jamais disponible, et le nombre de demandes émanant de studios commença à diminuer. Le Caine pensait que l'échec du projet était en grande partie de sa faute: "J'étais trop impressionné par le fait qu'il était peu souhaitable de "fixer" l'état de la "nouvelle musique" par trop fragile à cette étape, et j’ai sous-estimé les avantages d'offrir des appareils peu coûteux à d'éventuels utilisateurs ayant des objectifs très diversifiés 59 . 》

Un autre incident diminua considérablement la confiance de Le Caine en son instrument. Le 12 octobre 1961, il reçut un télégramme de Josef Tal: «Magnétophone arrivé sans encombre à Jérusalem, lettre suit exprimant remerciements. » Un mois plus tard arriva un second télégramme, indiquant que le magnétophone posait certains problèmes. Tal disait: «L'ingénieur veut savoir si l'appareil a été testé avant son expédition.» Le Caine écrivit immédiatement une lettre de cinq pages décrivant quelques méthodes d'essai permettant de déterminer si tous les boulons étaient serrés, si tous les câbles étaient bien fixés, et si les tubes à vide et les fusibles fonctionnaient. Comme le multi-track ne fonctionnait toujours pas après plusieurs semaines, Le Caine dut se rendre lui-même en Israël pour y réparer l'instrument. Accompagné de sa femme, il arriva à Jérusalem le 19 février 1962 et y resta deux semaines. Ils furent très bien reçus en Israël, furent conviés à des dîners officiels et à des visites de la ville, mais Le Caine ne prêta pratiquement pas

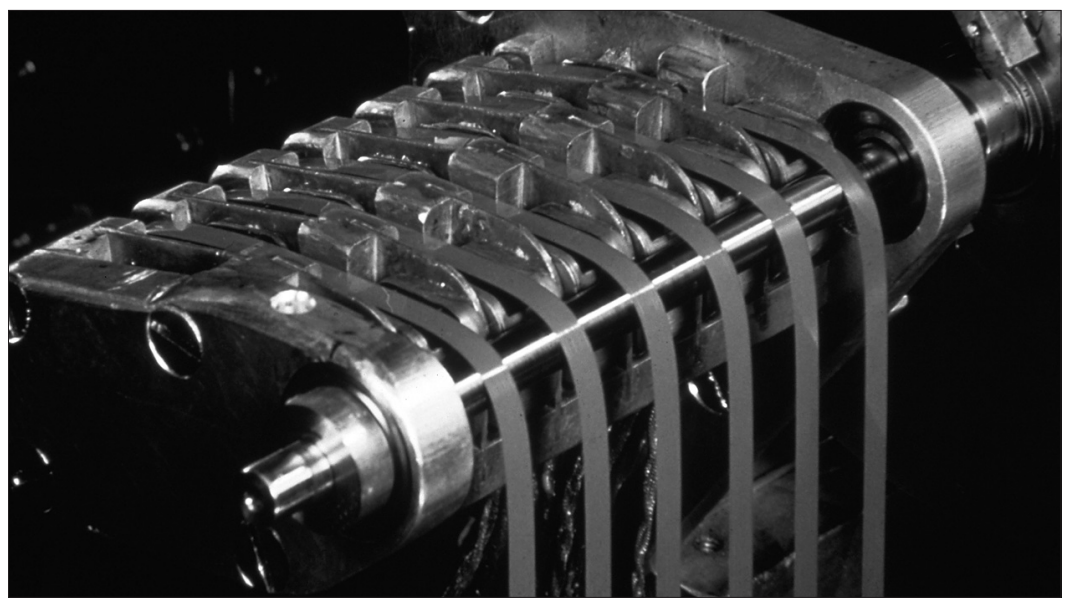

Six bandes stéréo défilent sur le même cabestan du magnétophone à application spéciale.

(C) Conseil national de recherches Canada. Reproduite avec la permission du ministre des Travaux publics et Services gouvernementaux Canada (2009) Source: Bibliothèque et Archives Canada/Fonds Hugh Le Caine. 
attention à ces événements. Il était extrêmement préoccupé par le multitrack, qu'il avait trouvé démonté sur le plancher d'une pièce presque vide qui constituait le studio de musique électronique. Une semaine après son arrivée, il envoya un télégramme au CNRC, indiquant que l'enregistreur marchait bien, mais que l'assistance technique au studio était quasi inexistante. Le Caine avait dû conclure des ententes spéciales pour que l'ingénieur soit libéré de ses obligations militaires durant sa visite ${ }^{60}$. De plus, malgré son enthousiasme pour l'instrument, il semble que Tal ne comprenait pas grand'chose aux aspects pratiques de son fonctionnement, n'avait que peu de patience pour les problèmes techniques, et était incapable d'aider l'ingénieur ou de le conseiller.

Le Caine fut affligé, non seulement par la pauvreté des installations à Jérusalem, mais également par une impression d'échec personnel. Il était découragé, car il avait l'impression de ne pas avoir été capable de fournir un outil opérationnel au studio de musique électronique. Même avant son voyage en Israël, il avait des doutes quant à l'utilisation du multipiste en musique électronique. $\mathrm{M}^{\mathrm{me}}$ Le Caine se souvient d'un incident qui eut lieu au moment où le multipiste fut envoyé en Israël:

Je commençais à être très frustrée par la manière dont les choses se passaient ici au Canada, par l'absence de publicité autour des travaux de Hugh et d'autres comme lui. J'ai dit à Hugh que s'il vivait aux États-Unis et que son multipiste était envoyé en Israël dans le cadre de l'Aide américaine d'urgence à Israël, il y aurait des conférences de presse, des articles dans les journaux, et le public saurait ce qui se passe et en serait fier. Je me souviens encore de Hugh, assis sur le bord de son lit, qui me répondit: «Bien sûr, mais avant de pavoiser, il faut d'abord avoir quelque chose à montrer.» Je n'en croyais pas mes oreilles. J'étais stupéfaite ${ }^{61}$.

En 1962, elle s'était habituée aux réticences de son mari à faire valoir ses travaux, mais elle était attristée par son refus d'admettre, ne serait-ce qu'à lui-même, la valeur de ce qu'il avait accompli. Elle voyait que les gens ne connaîtraient jamais ses meilleurs instruments si on ne leur en parlait pas, mais il restait obsédé par leurs imperfections. Trudi était particulièrement intéressée par l'orgue sensible au toucher, et tenta à plusieurs occasions d'aider Hugh à promouvoir l'instrument auprès de musiciens de sa connaissance. Néanmoins, lorsqu'elle l'incita à sortir l'orgue de sa «morgue» où il était remisé au CNRC et à le rapporter à la maison, il répliqua :

Je ne veux pas le voir à la maison. Je ne veux jamais le revoir. C'est impossible de le rapporter ici [...] Si l'orgue entre dans cette maison, je ne pourrai pas travailler à autre chose: je le démonterai et me dirai, je l'ai fait comme ceci, pourquoi ne pas essayer cette manière-là, et cela serait comme recommencer à nouveau à zéro. J'ai déjà résolu ce problème une fois !62
60. Le Caine à John Bowsher, 23 novembre 1961; Le Caine au CNRC (télégramme), 27 février 1962.

61. Entrevue, Trudi Le Caine, janvier 1983.

62. Ibid., octobre 1980. 